\title{
ON STATIONARY KINETIC SYSTEMS OF BOLTZMANN TYPE AND THEIR FLUID LIMITS
}

\author{
LEIF ARKERYD \\ Department of Mathematics, Chalmers University, S-41296 Gothenburg, Sweden \\ E-mail: arkeryd@math.chalmers.se
}

\begin{abstract}
The first part reviews some recent ideas and $L^{1}$-existence results for non-linear stationary equations of Boltzmann type in a bounded domain in $\mathbb{R}^{n}$ and far from global Maxwellian equilibrium. That is an area not covered by the DiPerna and P. L. Lions methods for the timedependent Boltzmann equation from the late 1980-ies.

The final part discusses the more classical perturbative case close to global equilibrium and corresponding small mean free path limits of fully non-linear stationary problems. Here the focus is on a particular two-rolls model problem including leading order hydrodynamic limits, but in a perspective of more general situations and the resolution of a variety of asymptotic stationary questions.
\end{abstract}

Remarks are made about stationary solutions as long-time limits of corresponding timedependent ones, and a number of open problems are also reviewed.

0. Introduction. In the first part I will review some recent ideas and existence results for stationary equations of Boltzmann type in a bounded domain in $\mathbb{R}^{n}$, an area not covered by the original DiPerna and P. L. Lions method for the time-dependent non-linear Boltzmann equation from the late 1980-ies. Their approach to existence fundamentally depends on conservation laws and entropy control to obtain a priori bounds and compactness properties. In the corresponding stationary problems, it is only the flows of such quantities that are under control, and they are not by themselves enough to imply all the required bounds and compactness related properties. But at least energy control is available from the moment flows. To replace an unavailable entropy bound, there is a weaker and more involved entropy dissipation control, and mass control may be forced onto the problem at a price. Using such devices and detailed properties of the collision geometry, together with A. Nouri in Marseille, we have developed an approach to sta-

2000 Mathematics Subject Classification: 76P05, 35Q35.

Key words and phrases: kinetic systems, fluid limits.

The paper is in final form and no version of it will be published elsewhere. 
tionary existence in an $L^{1}$-context for nonlinear Boltzmann related equations, also far from global Maxwellian equilibrium.

Before this, only the perturbative case close to global Maxwellian equilibria had been systematically studied, with Grad [G], Kogan [K] and Guiraud [Gu] (see also [H, P, UA]) as the pioneers in the late 1960-ies, and with the main arguments based on fixed points and contraction mapping techniques. But even there, mainly due to a lack of suitable estimates, up till now only little (but see [DEL, ELM]) has been done concerning the small mean free path limit of such fully nonlinear stationary problems. The final part of the survey will take up a possible remedy, consisting in suitable new techniques, again developed jointly with A. Nouri. I shall present this for a rotating two-rolls model problem including leading order hydrodynamic limits. Our expectation is that these techniques will ultimately resolve a variety of asymptotic stationary questions.

In the middle of the survey I will also comment on stationary solutions as longtime limits of the time-dependent development. A number of open problems will also be reviewed. For an introduction to the area see [C2, CIP].

1. On large data stationary existence. For the topic of large data stationary existence, our basic approach to the fully non-linear Boltzmann equation may be described as follows:

Velocities in the pair collisions of the Boltzmann equation- $-\left(v, v_{*}\right)$ (before) $\rightarrow\left(v^{\prime}, v_{*}^{\prime}\right)$ (after) - are connected by $\left(\sigma \in \mathcal{S}^{n-1}\right)$

$$
v^{\prime}=\frac{v+v_{*}}{2}+\frac{\left|v-v_{*}\right|}{2} \sigma, \quad v_{*}^{\prime}=\frac{v+v_{*}}{2}-\frac{\left|v-v_{*}\right|}{2} \sigma .
$$

The density of a rarefied gas is as usual modelled by nonnegative functions $f(x, v)$, with $x$ the position and $v$ the velocity. We shall write

$$
f(v)=f, f\left(v_{*}\right)=f_{*}, f\left(v^{\prime}\right)=f^{\prime}, f\left(v_{*}^{\prime}\right)=f_{*}^{\prime} .
$$

The $x$-domain $\Omega$ in position space is for convenience assumed smooth and strictly convex with inner normal $n(x)$ (but see the remarks further on).

On the ingoing boundary $\partial \Omega^{+}=\left\{(x, v) \in \partial \Omega \times \mathbb{R}^{n} ; v \cdot n(x)>0\right\}$ are given a diffuse reflection operator $\mathcal{R}$ and indata $f_{b}$. The boundary conditions are

$$
f=\Theta \mathcal{R} f+(1-\Theta) f_{b},
$$

where $0 \leq \Theta \leq 1$. The stationary Boltzmann equation in the domain $\Omega$ is

$$
\begin{aligned}
v \cdot \nabla_{x} f(x, v) & =Q(f, f)(x, v)=Q^{+}(x, v)-Q^{-}(x, v)=Q^{+}(x, v)-f \nu(f)(x, v) \\
& =\int_{\mathbb{R}^{3}} \int_{\mathcal{S}^{2}} B\left(v-v_{*}, \omega\right)\left[f^{\prime} f^{\prime *}-f f^{*}\right] d \omega d v_{*}, \quad x \in \Omega, v \in \mathbb{R}^{n},
\end{aligned}
$$

where $Q^{+}-Q^{-}$is the splitting into gain and loss parts of the collision operator $Q$, and $\nu$ is the collision frequency.

Integrating this boundary value problem multiplied with $\ln f$, gives a bound for the entropy dissipation,

$$
-e(f)=\int B\left(f f_{*}-f^{\prime} f_{*}^{\prime}\right) \ln \frac{f f_{*}}{f^{\prime} f_{*}^{\prime}}<c .
$$


If $f_{*}$ is bounded from below and $f^{\prime} f_{*}^{\prime}$ from above on sufficiently large sets, we get some control of possible mass concentrations for $f$ where $f$ is large. Of course this is more restricted than entropy, since e.g. the entropy dissipation is zero even for a Dirac type Maxwellian. But when it is applicable, as it in fact is for many stationary situations, then mass concentrations are prevented. It turns out that for stationary boundary values of type (1.1), this observation about the entropy dissipation is enough to deliver existence for the kinetic Povzner and Enskog equations in bounded domains in $\mathbb{R}^{n}$ [AN4], and for the Boltzmann equation in a slab both for soft and hard forces under no other restrictions than Grad's angular cut-off [AN3, AN6]. Let us discuss the slab case, and without loss of generality focus on a three dimensional velocity space. A typical result in the slab case is the following:

Let the slab be given by $-1 \leq x \leq 1$. Set $v=(\xi, \tilde{v})$, with $\xi$ parallel to $x$, and $\tilde{v}$ orthogonal to $x$. The stationary Boltzmann equation in the slab is

$$
\xi \frac{\partial}{\partial x} f(x, v)=Q(f, f)(x, v), \quad x \in[-1,1], v \in \mathbb{R}^{3} .
$$

For simplicity we take the kernel $B\left(v-v_{*}, \sigma\right)$ in the collision operator $Q$ as $\left|v-v_{*}\right|^{\beta} b(\theta)$, with

$$
-3<\beta<2, \quad b \in L_{+}^{1}(0, \pi), \quad b(\theta) \geq c_{2}>0 \text { a.e. }
$$

Given a constant $m>0$ and positive indata $f_{b}$ bounded away from zero on compacts, positive solutions $f$ to the slab equation(1.3) are sought such that

$$
\begin{gathered}
\int_{-1}^{1} \int_{\mathbb{R}^{3}}(1+|v|)^{\beta} f(x, v) d x d v=m, \\
f(-1, v)=k f_{b}(-1, v), \xi>0, \quad f(1, v)=k f_{b}(1, v), \xi<0,
\end{gathered}
$$

for some constant $k>0$. The constant $k$ is determined from the value $m$ of the $\beta$-norm (1.4). In this way, the lack of a mass estimate is compensated by forcing a $\beta$-norm control $m$ on the solution. If it were not for the problem with small velocities, the control (1.4) could be replaced by the condition $k=1$.

TheOREM 1.1 [AN3]. Suppose given $m>0,0 \leq \beta<2$, and indata $f_{b}$ satisfying

$$
\begin{gathered}
\int_{\xi>0}\left[\xi\left(1+|v|^{2}+\left|\ln f_{b}\right|\right)+(1+|v|)^{\beta}\right] f_{b}(-1, v) d v<\infty \\
\left.\int_{\xi<0}|\xi|\left(1+|v|^{2}+\left|\ln f_{b}\right|\right)+(1+|v|)^{\beta}\right] f_{b}(1, v) d v<\infty .
\end{gathered}
$$

Then there is a weak solution to the stationary slab problem (1.3-5).

Analogous results hold for boundary conditions of diffuse reflection type, (i.e. (1.1) with $\Theta=1$ ) and for the mixed case. There are similar theorems for soft forces, i.e. for $0>\beta>-3$. In all those cases, the collision frequency integral along characteristics, essentially behaves like a volume integral, which is a priori controlled in the approximation scheme. It is a serious obstacle that this is not so for the nonlinear Boltzmann equation itself in higher dimensions $(n>1)$,

$$
v \cdot \nabla_{x} f(x, v)=Q(f, f), \quad x \in \Omega, v \in \mathbb{R}^{n} .
$$


However, that problem can be overcome, at least as long as the other main obstacle to the full $\mathbb{R}^{n}$-result is eliminated, namely the small velocities in the nonlinear collision operator. So consider $Q$ given by

$$
\int_{\mathbb{R}^{n}} \int_{S^{n-1}} \chi_{s}\left(v, v_{*}, \sigma\right) B\left(v-v_{*}, \sigma\right)\left(f\left(x, v^{\prime}\right) f\left(x, v_{*}^{\prime}\right)-f(x, v) f\left(x, v_{*}\right)\right) d v_{*} d \sigma
$$

with $s>0$, and

$$
\chi_{s}\left(v, v_{*}, \sigma\right)=0 \text { if }|v|<s \text { or }\left|v_{*}\right|<s \text { or }\left|v^{\prime}\right|<s \text { or }\left|v_{*}^{\prime}\right|<s, \chi_{s}\left(v, v_{*}, \sigma\right)=1 \text { else. }
$$

The removal of small velocities through $\chi_{s}$, again allows mass to be estimated by a priori controlled energy, and we may study the equation with given indata instead of the previous $\beta$-norm $m$ plus indata profile. So given a function $f_{b}>0$ defined on $\partial \Omega^{+}$, we look for a solution $f$ to $(1.2)$ with

$$
f(x, v)=f_{b}(x, v), \quad(x, v) \in \partial \Omega^{+} .
$$

A priori estimates along characteristics using the exponential solution form, together with new local information from the entropy dissipation control, leads to the following result.

THEOREM 1.2 [AN7]. Suppose that $f_{b}>a e^{-d v^{2}}$ for some $a, d>0$ and a.a. $(x, v) \in \partial \Omega^{+}$, and that

$$
\int_{(x, v) \in \partial \Omega^{+}}\left[v \cdot n(x)\left(1+v^{2}+\ln ^{+} f_{b}(x, v)\right)+1\right] f_{b}(x, v) d x d v<\infty .
$$

Then the equation (1.6) has a solution satisfying the boundary condition (1.7).

REMARKS. If we were to keep the small velocities and remove the truncation $\chi_{s}$, a variant of the limiting procedure in the proof would still work but, besides admitting the desired solution of the boundary value problem, would also allow the unwanted alternative of a total collapse as Dirac measure at velocity zero, which we don't know how to prevent. The removal of the small-velocity $\chi_{s}$-truncation probably requires fresh ideas. Mathematically the imposed small velocity cut-off is a serious restriction, but physically less so, if e.g. the velocity is only removed below some Planck scale. Physically more serious is the lack of uniqueness (or at least local uniqueness i.e. isolated solutions), a problem $(\mathcal{P}:)$ of course shared with the $[\mathrm{DPL}]$ time dependent theory in its present state.

$\mathcal{P}$ : The technical restrictions on $\Omega$ and $f_{b}$ can be relaxed. In fact we even expect the result to hold for the same mathematically and physically natural, non-smooth domains as in the time dependent case $[\mathrm{AH}]$, namely with boundaries having finite Hausdorff measure plus a certain cone condition.

I will end this first part of the presentation with two interesting technical points from the proofs. It is clear from what I have already said, that the behaviour at small velocities is a major difficulty for stationary kinetic problems. Let us first see how the small velocities can be handled for the slab problem with given indata profile. Actually, for the slab this difficulty comes up as the small velocity in the slab direction, $\xi$. Let us focus on one instance of the main idea, which is to deduce the small velocity behaviour from the large velocity behaviour. To minimize technicalities, assume Maxwellian forces, 
i.e. $\beta=0$. We start from the collision operator with an extra velocity-cutoff for small $\xi$,

$$
Q^{s}(f, f)(x, v)=\int_{\mathbb{R}^{3}} \int_{\mathcal{S}^{2}} \chi_{s} B(\sigma)\left[f^{\prime} f^{\prime *}-f f^{*}\right] d \sigma d v_{*} .
$$

In the limit of disappearing cut-off, $s \rightarrow+0$, we then recover the desired Maxwellian collision operator with only a Grad angular cut-off.

We also start from an approximation of the equation including an extra absorption,

$$
s f^{s}+\xi \frac{\partial}{\partial x} f^{s}=Q^{s}\left(f^{s}, f^{s}\right), \quad x \in \Omega, v \in \mathbb{R}^{n} .
$$

Since $\beta=0$, the moment condition (1.4) fixes the mass. The boundary values are given indata profile (1.5). This approximation can be solved by fixed point arguments and devices related to the corresponding time dependent problem. They deliver a solution with the constant $k$ of (1.5) bounded over the approximations,

$$
0<\inf _{S} k_{s} \leq \sup _{S} k_{s}<\infty
$$

In the usual way of multiplying the equation with $\xi$ and integrating, the $\xi^{2}$-moment is uniformly controlled in $s$ and $x$,

$$
\int \xi^{2} f^{s} d v \leq c_{3}<\infty
$$

and the entropy dissipation is likewise uniformly controlled in $s$,

$$
-e\left(f^{s}\right):=-\int Q^{s}\left(f^{s}, f^{s}\right) \ln f^{s} d x d v \leq c_{4}<\infty .
$$

From here the small- $\xi$ control follows by the geometry, as I shall illustrate for the case when $|\tilde{v}|>10 \lambda$ and $\lambda \gg 10$. Take $v_{*}=\left(\xi_{*}, \tilde{v}_{*}\right)$ with $\left|\tilde{v}_{*}\right| \leq 10$, and $10^{-1} \leq\left|\xi_{*}\right| \leq 1$. The exponential form of the equation immediately gives for all such $v_{*}$ and all $s, x$, that $f^{s}\left(x, v_{*}\right) \geq c_{5}>0$. For these $\left(x, v, v_{*}, \sigma, s\right)$ and for $L>2$, it holds that

$$
c_{2} c_{5} f^{s}(x, v) \leq b(\theta) f^{s}(x, v) f^{s}\left(x, v_{*}\right) \leq L b(\theta) f^{\prime s} f_{*}^{\prime s}+\frac{2}{\ln L} b(\theta)\left(f^{s} f_{*}^{s}-f^{\prime s} f_{*}^{\prime s}\right) \ln \frac{f^{s} f_{*}^{s}}{f^{\prime s} f_{*}^{\prime s}} .
$$

And so

$$
\int_{s \leq|\xi| \leq 1,10 \lambda \leq|\tilde{v}|} f^{s}(x, v) d x d v \leq \frac{c L}{\lambda^{4}}+\frac{c}{\ln L} .
$$

This is arbitrarily small for $L$ sufficiently large, and then $\lambda$ taken large enough.

Let me also say a few words about the ideas behind the $n D$-result with given indata in Theorem 1.2. Without loss of generality we can again restrict the discussion to dimension $n=3$. As in the previous slab case, the first step in the proof is to solve the equation with an extra absorption term $\alpha f$ added. We start from the weak form of the equation,

$$
\begin{aligned}
& \int_{\Omega \times \mathbb{R}^{3}}\left[-\alpha f^{\alpha}+f^{\alpha} v\right.\left.\nabla x+Q\left(f^{\alpha}, f^{\alpha}\right)\right] \varphi(x, v) d x d v \\
&=-\int_{\partial \Omega^{+}} v \cdot n(x) f_{b} \varphi(x, v) d x d v-\int_{\partial \Omega^{-}} v \cdot n(x) f^{\alpha} \varphi(x, v) d x d v .
\end{aligned}
$$

The collision integral $\int Q(f, f) \varphi d v$ vanishes for $\varphi=1, v, v^{2}$, and is non-positive for $\varphi=$ $\ln f$. That leads to a priori $\alpha$-dependent estimates of mass $\int f$, energy $\int f v^{2}$, and entropy 
$\int f \ln f$. Using fixed point arguments and other devices just like the slab case, it follows that the $\alpha$-approximation has a non-negative solution $f^{\alpha}$.

Again using the weak form, we can estimate outgoing mass flow a priori by ingoing mass flow independently of $\alpha>0$. The exponential form of the equation is

$$
\begin{aligned}
f^{\alpha}(x, v)= & f_{b}\left(x-s^{+}(x, v) v, v\right) e^{-\int_{-s^{+}(x, v)}^{0}\left(\alpha+\nu\left(f^{\alpha}\right)(x+s v, v)\right) d s} \\
& +\int_{-s^{+}(x, v)}^{0} Q^{+}\left(f^{\alpha}, f^{\alpha}\right)(x-\tau v, v) e^{-\int_{-\tau}^{0}\left(\alpha+\nu\left(f^{\alpha}\right)(x+t v, v)\right) d t} d \tau
\end{aligned}
$$

Here $s^{+}$is the time it takes to reach the ingoing boundary point along the characteristic $(x-s v, v)$. It follows that

$$
f_{b} e^{-\int \nu} \leq f(x, v) \leq f_{\text {outgoing }} e^{\int \nu},
$$

and so the exponential form gives uniform estimates of $f^{\alpha}$ along characteristics outside a small set; given $\epsilon>0$ there is a constant $C_{\epsilon}$ independent of $\alpha$, so that outside a set (depending on $\alpha$ ) of characteristics of measure $\epsilon$, it holds that $f^{\alpha}<C_{\epsilon}$. We replace $f^{\alpha}$ by zero outside the nicely bounded characteristics. Then the weak $\operatorname{limit} f_{\epsilon}=w-\lim f_{\text {restr }}^{\alpha}$ increases with $1 / \epsilon$.

With the final limit $f=s$-lim $f_{\epsilon}$ of these approximate solutions a rather naive candidate for the true solution, the hard part is to prove that this candidate really solves the desired problem. We use the so-called iterated integral form of the equation, where it is easy to suppress the solution along whole characteristics, by setting the test function equal to zero along them,

$$
\begin{aligned}
\int_{\partial \Omega^{+}} & \left(f_{b} \varphi\right)(x, v) v \cdot n(x) d x d v \\
& \quad+\int_{\partial \Omega^{-}}\left(\int_{-s^{+}(x, v)}^{0}\left[-\alpha f \varphi+Q(f, f) \varphi+f v \cdot \nabla_{x} \varphi\right](x+\sigma v, v) d \sigma\right)|v \cdot n(x)| d x d v=0
\end{aligned}
$$

The iterated collision integral is well defined through our approximation scheme, even if $\mathrm{Q}$ may not be integrable. The replacement of the test functions by zero along certain characteristics is possible, since the test functions are in $L^{\infty}$, and only required to be differentiable along characteristics.

The main difficulty with this removal procedure, is the following. Consider the collision frequency $\nu=\int d \sigma \int B f_{*}^{\alpha} d v_{*}$. It may happen at a point $x \in \Omega$ along a retained characteristic for $f^{\alpha}$, that other characteristics through the point $x \in \Omega$ are not retained. This may decrease the collision frequency at $x$, which is an integral in the second velocity variable $v_{*}$. The second step in the proof consists in a study of the interaction between what is retained and what is removed. A central part is a lemma quantifying in what sense the possibly bad behaviour along the particular small set of removed characteristics, in the limit does not influence the behaviour based on the rest of phase space, in spite of the mixing non-linear character of the collision operator. The proof of this lemma involves some violent scalings and estimates. Finally the third step in the proof is a check that the final $L^{1}$-limit of the approximations, by itself solves the boundary value problem. 
2. On long time behaviour. Stationary solutions to the Boltzmann equation, besides having an intrinsic interest, come up as natural candidates for the time asymptotics of corresponding evolutionary problems after the transients have died down. Rigorous convergence results in various topologies for the limit of infinite time are known, when the boundary conditions are periodic, or specular reflections as well as diffuse reflections with temperature and pressure constant around the boundary. But this is also an area with many important (cf [CCW, CGT, V, W]) and still open problems of varying difficulty.

$\mathcal{P}$ : In the long term, does one always find convergence to a steady situation, or are also periodic and more irregular types of limiting behaviour possible?

$\mathcal{P}$ : When there is convergence, what is its type and, when meaningful, its rate?

There are deep and interesting ongoing activities about this rate problem by Villani and Desvillettes, which I will not enter. I will only illustrate the area of time asymptotics with an easily explained case of strong $L^{1}$-convergence to a unique Maxwellian, essentially controlled by the boundary interaction. Consider the time dependent equation

$$
\left(\partial_{t}+v \cdot \nabla_{x}\right) f=Q(f, f), \quad t \in \mathbb{R}^{3}, x \in \Omega, v \in \mathbb{R}^{3},
$$

where $\Omega$ is bounded, strictly convex and smooth, and $Q$ as before denotes the Boltzmann collision operator, together with an initial condition

$$
f(0, x, v)=f_{0}(x, v), \quad x \in \Omega, v \in \mathbb{R}^{3} .
$$

Here $f_{0}$ has finite mass, energy, and entropy. Let us assume Maxwellian diffuse reflection on the boundary,

$f(t, x, v)=M(\xi) \int_{v^{\prime} \cdot n(x)<0}\left|v^{\prime} \cdot n(x)\right| f\left(t, x, v^{\prime}\right) d v^{\prime}, \quad t \in \mathbb{R}^{+}, x \in \partial \Omega, v \cdot n(x)>0$

with

$$
M(v)=c_{0} \exp \left(-.5 \theta|v|^{2}\right)
$$

a normalized Maxwellian, $c_{0}$ a normalization constant, and $\frac{1}{\theta}>0$ a constant temperature. The relevant equilibrium solution is $f_{s}=c_{1} M$ with

$$
c_{1}=\frac{\int_{\Omega \times \mathbb{R}^{3}} f_{0}(x, v) d x d v}{\int_{\Omega \times \mathbb{R}^{3}} M(v) d x d v} .
$$

The following existence result holds by the time-dependent existence theory:

TheOREM 2.1 [AM]. There exists a mild solution

$$
f \in C\left(\mathbb{R}^{+}, L^{1}\left(\Omega \times \mathbb{R}^{3}\right)\right), \quad f \geq 0,
$$

to the initial boundary value problem (2.1-3).

After multiplication with $\ln \frac{f}{M}$, the equation gives

$$
\left(\partial_{t}+v \cdot \nabla_{x}\right)\left(f \ln \frac{f}{M}\right)=Q(f, f) \ln \frac{f}{M}+Q(f, f) .
$$

Integrating this over $[0, t] \times \Omega \times \mathbb{R}^{3}$ and using the initial and boundary values, implies 


$$
\begin{aligned}
& \int_{\Omega \times \mathbb{R}^{3}}\left(f \ln \frac{f}{M}\right)(t, x, v) d x d v-\int_{0}^{t} \int_{\partial \Omega \times \mathbb{R}^{3}} v \cdot n(x)\left(f \ln \frac{f}{M}\right) d \tau d x d v \\
&-\frac{1}{4} \int_{0}^{t} \int_{\Omega \times \mathbb{R}^{3}} e(f) d \tau d x d v \leq \int_{\Omega \times \mathbb{R}^{3}} f_{0} \ln \frac{f_{0}}{M}(x, v) d x d v .
\end{aligned}
$$

Since $e(f) \leq 0$ and the boundary integral is non-positive by Darrozes \& Guiraud's inequality (see [C2]), it follows that

$$
\int_{\Omega \times \mathbb{R}^{3}} f \ln \frac{f}{M}(t, x, v) d x d v<c,
$$

and

$$
0 \leq \int_{0}^{+\infty} \int_{\Omega \times \mathbb{R}^{3}} e(f)(t, x, v) d t d x d v<c .
$$

The density $f$ is a Maxwellian, when the integrand in $e$ is zero a.e.. And the desired convergence to a Maxwellian is obtained by an analysis of how $f$ is close to a Maxwellian, when the integral of $e$ for large times is close to zero. Once the limit is proved to be Maxwellian, the limit boundary condition by itself turns out to select (via Green's identity or directly) the precise limit Maxwellian. This leads to the following convergence result.

THEOREM 2.2 [AN1]. Let $f$ be a solution of the initial boundary value problem (2.1-3) with nowhere vanishing collision kernel. When $t$ tends to infinity, $f(t, .,$.$) converges$ strongly in $L^{1}\left(\Omega \times \mathbb{R}^{3}\right)$ to the global Maxwellian $c_{1} M$.

In the theorem, $M$ comes from the boundary condition (2.3), and $c_{1}$ is given by the conservation of mass $\left(c_{1}=\int f_{0} / \int M\right)$.

Specific for the kinetic case, and not generally correct in fluid dynamics situations, the natural restrictions on the domain are few, only that the boundary has finite $(n-1)$ dimensional Hausdorff measure - for reasonable traces to exist - and obeys a certain cone condition - to ensure that a molecule which falls on the surface has a strictly positive probability to be reflected into some body angle of size (uniformly over the surface) bounded from below. Theorem 2.2 can be generalized to that natural type of boundary. Also the Maxwellian is uniquely determined by the initial value and the boundary condition.

$\mathcal{P}$ : The uniqueness in Theorem 2.2 has so far not been proved for the cases of periodic, specular, or direct reflection boundary conditions, where some limit existence results are known. What can rigorously be said about uniqueness in those cases? Can anything be said about convergence when the boundary temperature in the above problem is varying?

3. The two-rolls model and fluid limits. Stationary solutions are also of importance in rarefied gas dynamics, which deals with gas phenomena, where Navier-Stokes type equations are not valid in some significant region of the flow field. A useful parameter is the Knudsen number $K n$, the ratio of the molecular mean free path (in ordinary air $\sim 10^{-5} \mathrm{~cm}$ ) to a typical length scale for the flow. This length scale could be based on the gradients occurring in the flows. Often the regions are very thin, where deviation from the Navier-Stokes behaviour is expected, and the non Navier-Stokes terms become important. The broad picture is one of normal regions where the gas flow follows the 
macroscopic fluid equations, plus thin shock layers, boundary layers, and initial layers, where matching conditions are sought between fluid regions on each side of the shock, or between outside initial or boundary control and interior fluid behaviour.

From that picture, I will now focus on a stationary two-rolls problem with boundary layers, and its behaviour in the small $K n$ limit. The set-up is as follows. Consider the stationary Boltzmann equation in the space $\Omega$ between two coaxial cylinders. Denoting by $(r, \theta, z)$ and $\left(v_{r}, v_{\theta}, v_{z}\right)$ respectively, the cylindrical spatial coordinates and the corresponding velocity coordinates, for parameter ranges where the system stays axially and rotationally uniform, the solutions are thus positive functions $f\left(r, v_{r}, v_{\theta}, v_{z}\right)$. In these coordinates the Boltzmann equation may be written

$$
v_{r} \frac{\partial f}{\partial r}+\frac{1}{r} N f=\frac{1}{\epsilon^{j}} Q(f, f), \quad r \in\left(r_{A}, r_{B}\right), \quad\left(v_{r}, v_{\theta}, v_{z}\right) \in \mathbb{R}^{3} .
$$

Here

$$
N f:=v_{\theta}^{2} \frac{\partial f}{\partial v_{r}}-v_{\theta} v_{r} \frac{\partial f}{\partial v_{\theta}} .
$$

The Knudsen number $\epsilon^{j}=K n$ will in this talk be taken as $j=4$. As boundary conditions, functions $f_{b}$ are given on the ingoing boundary $\partial \Omega^{+}$, i.e. $\left\{\left(r_{A}, v\right) ; v_{r}>0\right\}$ and $\left\{\left(r_{B}, v\right) ; v_{r}<0\right\}$. For convenience we take them as Maxwellians $M_{\alpha}$ having the known values at the boundary for pressure $P_{\alpha}$, temperature $T_{\alpha}$, and rotation rate $v_{\theta \alpha}$, where $\alpha=A$ or $B$. We may assume that the solutions are even in the $v_{z}$-variable. The earlier mentioned slab existence without small velocity cut-off in one space dimension, can actually be extended to this problem with space dimension two, namely

THEOREM 3.1 [AN9]. Given $m=\int_{r_{A}}^{r_{B}} \int_{\mathbb{R}^{3}}(1+|v|)^{\beta} f d x d v$ and boundary Maxwellians, there exists a weak $L^{1}$-solution to the Boltzmann equation for hard forces in the two-roll domain with $\beta$-moment $m$ and the given indata profile (much more general profiles are possible).

As in the slab case discussed earlier, the proof is based on weak $L^{1}$ compactness and does not require any cut-off for small velocities. It gives on the other hand no information about uniqueness, isolated solutions, fluid limits with extra terms, or possible ghost effects. Such results still have to be based on the asymptotic methods inititated by Grad $[\mathrm{G}]$, Kogan $[\mathrm{K}]$ and Guiraud [Gu] a full generation ago. Many important problems are still open - at least when it comes to rigorous mathematical analysis, in contrast to formal asymptotics and scientific computing, which has matured much further. For the formal and numerical aspects, a recent monograph by Y. Sone [S] from Kyoto gives a good picture, and the 1993 monograph by N. Maslova $[\mathrm{M}]$ is a fine introduction to what was known about the rigorous mathematics.

I will now devote the rest of the survey to asymptotic problems for the rotating two-roll situation, and in particular some recent progress by AN and myself concerning rigorous results for bifurcating multiple solutions and their positivity. Expand the solution to (3.1) as $f=M\left(1+\psi+\epsilon^{j_{0}} R\right)$ with

$$
\psi=\sum_{1}^{j_{0}} \epsilon^{j} \psi^{j}, \quad M=(2 \pi)^{-\frac{3}{2}} \exp \left(-\frac{v^{2}}{2}\right),
$$


and split the rest term as

$$
R=P_{0} R+\left(I-P_{0}\right) R=R_{\|}+R_{\perp},
$$

where $P_{0}$ is the orthogonal projection on the hydrodynamic part. Here $\sum_{1}^{j_{0}} \epsilon^{j} \psi^{j}$ is the asymptotic expansion with boundary values of the terms equal to the corresponding order in the $\epsilon$-expansions of the boundary Maxwellians $M_{\alpha}$. We take $j_{0}=4$, and assume that the rotational velocities of the inner and outer cylinders are scaled as $u_{\theta A}=\epsilon u_{\theta A 1}$ and $u_{\theta B}=\epsilon u_{\theta B 1}$ respectively, and that the non-dimensional temperature difference is

$$
\tau_{B}=\epsilon^{2} \tau_{B 2},
$$

where $u_{\theta A 1}, u_{\theta B 1}, \tau_{B 2}$ are given. The asymptotic expansion in $\epsilon$ per se, can be computed based on a splitting into interior Hilbert behaviour, together with boundary layers of suction and Knudsen type (cf [AN2, AN5, BCN, BU, C1, GPS, GP, V]). That expansion is of course not by itself a density solution of the Boltzmann equation, since it satisfies the Boltzmann equation only up to some order - in our case $\epsilon^{4}$ - and may by its essentially polynomial character become negative, whereas a real density should be everywhere positive. Also, mathematically interesting but not implied by the formal asymptotics, is in what sense the leading order gas dynamics equations are limits of the kinetic ones. An important problem is here a rigorous study of the rest term, using as ingoing boundary values what remains of the boundary Maxwellians after the asymptotic expansion. To obtain our bifurcation situation, we assume that the temperature and density are coupled by

$$
\omega_{B}=\frac{\epsilon^{2}}{1+\epsilon^{2} \tau_{B 2}}\left(\frac{r_{B}^{2}-1}{r_{B}^{2}} u_{\theta A 1}^{2}-\tau_{B 2}+\Delta \epsilon\right),
$$

where $\Delta$ is a parameter. It then holds for the full solution $f$ that

TheOREM 3.2 [AN8]. Assume that $\left(u_{\theta A 1}-u_{\theta B 1} r_{B}\right)\left(3 u_{\theta A 1}+u_{\theta B 1} r_{B}\right)>0$. Then there is a negative $\Delta_{\text {bif }}$, such that for $\Delta<\Delta_{\text {bif }}$ and $0<\epsilon$ small enough, there are two positive, isolated non-negative $L^{1}$-solutions $f_{\epsilon}^{j}, j=1,2$ of the Boltzmann equation in the two-rolls domain (3.1), with Maxwellian indata (1.7), for which

$$
\int M^{-1} \operatorname{essips}_{r \in\left(r_{A}, r_{B}\right)}\left|f_{\epsilon}^{j}(r, v)\right|^{2} d v<+\infty .
$$

The two solutions have different outward radial bulk velocities of order $\epsilon^{3}$. For fixed $\epsilon$, they converge to the same solution when $\Delta$ increases to $\Delta_{b i f}$. The solutions have rigorous leading order hydrodynamic limits when $\epsilon \rightarrow 0$.

The monograph I mentioned earlier by Sone $[\mathrm{S}]$ from Kyoto already contains this result on the level of formal asymptotic expansions and numerics. It also resolves many other situations besides this bifurcation for the two-rolls problem. In all those cases there is a corresponding version of Theorem 3.2. In particular this proves rigorously the existence in some cases even of three or more simultaneous positive solutions to the same stationary boundary value problem for the two-rolls system.

$\mathcal{P}$ : Some of the stationary numerical results by the Kyoto group, were obtained as time asymptotics of corresponding time-dependent problems. So here again comes the impor- 
tant mathematical question, whether a time dependent solution really converges to a stationary one with time, when the temperature and pressure are varying over the boundary. The three simultaneous stationary solutions we rigorously obtained, are a warning about possible complications. Since stationary solutions are sometimes not unique in the nonlinear case, how is that coupled to the time-asymptotics?

The proof of Theorem 3.2 requires a study of the equation for the rest term $R$, to which we now turn. $R$ should be a solution to

$$
v_{r} \frac{\partial R}{\partial r}+\frac{1}{r} N R=\frac{1}{\epsilon^{4}}\left(\tilde{L} R+2 \tilde{J}(R, \bar{\chi} \psi)+\epsilon^{4} \tilde{J}(R, R)+l\right),
$$

where

$$
l=\frac{1}{\epsilon^{4}}\left(\tilde{L}(\bar{\chi} \psi)+\tilde{J}(\bar{\chi} \psi, \bar{\chi} \psi)-\epsilon^{4} D(\bar{\chi} \psi)\right),
$$

$\tilde{J}$ is the rescaled quadratic Boltzmann collision operator,

$\tilde{J}(\Phi, \psi)(v)$

$:=\frac{1}{2} \int_{\mathbb{R}^{3} \times S^{2}} B\left(v-v_{*}, \omega\right) M\left(v_{*}\right)\left(\Phi\left(v^{\prime}\right) \psi\left(v_{*}^{\prime}\right)+\Phi\left(v_{*}^{\prime}\right) \psi\left(v^{\prime}\right)-\Phi\left(v_{*}\right) \psi(v)-\Phi(v) \psi\left(v_{*}\right)\right) d v_{*} d \omega$, and $\tilde{L}$ is the operator $\tilde{J}$ linearized around the Maxwellian,

$$
(\tilde{L} \Phi)(v):=\frac{1}{\epsilon^{4}} \int_{\mathbb{R}^{3} \times S^{2}} B\left(v-v_{*}, \omega\right) M\left(v_{*}\right)\left(\Phi\left(v^{\prime}\right)+\Phi\left(v_{*}^{\prime}\right)-\Phi\left(v_{*}\right)-\Phi(v)\right) d v_{*} d \omega=\tilde{K}(\Phi)-\tilde{\nu} \Phi .
$$

Our a priori estimates for $R$ that underlie the contraction mappings leading to Theorem 3.2 , are uniform in $\epsilon$, so the low order hydrodynamic limits can be established in a mathematically rigorous way. Denoting the right hand side of the rest-term equation (3.2) by $\frac{\tilde{L} R+g}{\epsilon^{4}}$, Green's formula gives that

$$
-\int_{\Omega \times \mathbb{R}^{3}} M R \tilde{L} R \leq \int_{\Omega \times \mathbb{R}^{3}} M g R+\epsilon^{4} \int_{\partial \Omega^{+}} M R_{b}^{2} .
$$

Since $c \int_{\Omega \times \mathbb{R}^{3}} \tilde{\nu} M R_{\perp}^{2} \leq-\int_{\Omega \times \mathbb{R}^{3}} M R \tilde{L} R$, and since $g$ is essentially nonhydrodynamic, it is actually enough to consider $g=g_{\perp}$, and we may conclude that

$$
\int_{\Omega \times \mathbb{R}^{3}} \tilde{\nu} M R_{\perp}^{2} \leq c \int M g^{2}+\epsilon^{4} \int_{\partial \Omega^{+}} M R_{b}^{2}
$$

As for the hydrodynamic estimates, fairly exact computations are required, and here I have to refer to our paper.

$\mathcal{P}$ : These techniques should also hold the key to resolving many other problems. Work is in progress [AN10] on a similar study about the axially inhomogeneous Taylor Couette problem of [SD1]. What can be done in this direction for the Bénard problem [SD2], for multi-component gases [ATT], for ghost effects, for obtaining fluid bifurcations from kinetic ones, etc.

I would like to end with a discussion of the positivity proof for our solutions of asymptotic expansion type $f=M\left(1+\psi+\epsilon^{j_{0}} R\right)$. Start for positivity from the related problem

$$
\begin{aligned}
& v \cdot \nabla_{x} f=Q\left(f^{+}, f^{+}\right)-M L\left(M^{-1} f^{-}\right), \quad(x, v) \in \Omega \times \mathbb{R}^{3}, \\
& f=f_{b}, \quad \text { on } \partial \Omega^{+}
\end{aligned}
$$


where $f^{+}=\max \{f, 0\}$ and $f^{-}=\max \{-f, 0\}$. Also for this equation there are solutions which can be expanded as $f=M\left(1+\bar{\chi} \sum_{i=1}^{4} \epsilon^{i} \psi_{i}+\epsilon^{4} R\right)$, and solved like the previous problem. Proving that $f^{-}=0$ will imply that the rest terms of the two expansions coincide, and that the originally obtained solution $f$ is nonnegative and isolated.

THEOREM 3.3 [AN10]. Let $\Omega$ be a bounded set in $\mathbb{R}^{3}$, and $f_{b}$ a nonnegative function defined on $\partial \Omega^{+}$. If a function $f$ such that $M^{-1} f \in \tilde{L}^{\infty}\left(\Omega \times \mathbb{R}^{3}\right)$ satisfies

$$
\begin{aligned}
& v \cdot \nabla_{x} f=Q\left(f^{+}, f^{+}\right)-M L\left(M^{-1} f^{-}\right), \quad(x, v) \in \Omega \times \mathbb{R}^{3}, \\
& f=f_{b}, \quad \text { on } \partial \Omega^{+},
\end{aligned}
$$

then $f^{-}=0$ and $f=f^{+}$solves the boundary value problem

$$
\begin{aligned}
& v \cdot \nabla_{x} f=Q(f, f), \quad \Omega \times \mathbb{R}^{3}, \\
& f=f_{b}, \quad \text { on } \partial \Omega^{+} .
\end{aligned}
$$

Proof of Theorem 3.3. The function $F=M^{-1} f$ satisfies

$$
v \cdot \nabla_{x} F=J\left(F^{+}, F^{+}\right)-L\left(F^{-}\right), \quad F=M^{-1} f_{b}, \quad \text { on } \partial \Omega^{+} .
$$

Define $J^{+}$and $J^{-}$by $J(\varphi, \varphi)=J^{+}(\varphi, \varphi)-J^{-}(\varphi, \varphi)$, where

$$
\begin{aligned}
& J^{+}(\varphi, \varphi)(v):=\int\left|v-v_{*}\right|^{\beta} b(\theta) M_{*} \varphi^{\prime} \varphi_{*}^{\prime} d v_{*} d \omega, \\
& J^{-}(\varphi, \varphi)(v):=\varphi(v) \int\left|v-v_{*}\right|^{\beta} b(\theta) M_{*} \varphi_{*} d v_{*} d \omega .
\end{aligned}
$$

Also, $F^{-}$satisfies

$$
\begin{aligned}
& -v \cdot \nabla_{x} F^{-}=\chi_{F^{-} \neq 0}\left(J^{+}\left(F^{+}, F^{+}\right)-L\left(F^{-}\right)\right), \\
& F^{-}=0, \quad \text { on } \partial \Omega^{+} .
\end{aligned}
$$

Multiplying (3.3) by $-M F^{-}$, integrating on $\Omega \times \mathbb{R}^{3}$ and using that

$$
-\int M F^{-} \chi_{F^{-} \neq 0} L\left(F^{-}\right) d v=-\int M F^{-} L\left(F^{-}\right) d v \geq c \int M \tilde{\nu}\left|\left(I-P_{0}\right) F^{-}\right|^{2} d v,
$$

implies that

$$
\int_{\partial \Omega^{-}}|v \cdot n| M\left(F^{-}\right)^{2}+c \int_{\Omega \times \mathbb{R}^{3}} M \tilde{\nu}\left|\left(I-P_{0}\right) F^{-}\right|^{2} \leq-\int M F^{-} \chi_{F^{-} \neq 0} J^{+}\left(F^{+}, F^{+}\right) \leq 0 .
$$

It follows that

$$
F^{-}=0, \text { on } \partial \Omega^{-}, \quad L\left(F^{-}\right)=0 .
$$

And so, $F^{-}$satisfies

$$
F^{-}=0 \text {, on } \partial \Omega^{-} \cup \partial \Omega^{+}, \quad v \cdot \nabla_{x} F^{-} \leq 0 .
$$

This implies that $F^{-}$is identically zero.

Acknowledgements. The research was carried out within the program of the HYKE network under European Union contract HPRN-CT-2002-00282. 


\section{References}

[ATT] K. Aoki, S. Takata and S. Taguchi, Vapor flows with evaporation and condensation in the continuum limit: effect of a trace of noncondensable gas, preprint, 2002.

$[\mathrm{AC}] \quad$ L. Arkeryd and C. Cercignani, On the convergence of solutions of the Enskog equation to solutions of the Boltzmann equation, Comm. Part. Diff. Eqns. 14 (1989), 1071-1089.

$[\mathrm{AH}] \quad$ L. Arkeryd and A. Heintz, On the solvability and asymptotics of the Boltzmann equation in irregular domains, Comm. Part. Diff. Eqns. 22 (1997), 2129-2152.

[AM] L. Arkeryd and N. Maslova, On diffuse reflection at the boundary for the Boltzmann equation and related equations, J. Stat. Phys. 77 (1994), 1051-1077.

[AN1] L. Arkeryd and A. Nouri, Boltzmann asymptotics with diffuse reflection boundary conditions, Monatshefte für Math. 123 (1997), 285-298.

[AN2] L. Arkeryd and A. Nouri, A condensation-evaporation problem, SIAM J. Math. Anal. 29 (1998), 30-47.

[AN3] L. Arkeryd and A. Nouri, The stationary Boltzmann equation in the slab with given weighted mass for hard and soft forces, Ann. Scuola Norm. Sup. Pisa Cl. Sci. 27 (1998), $533-556$.

[AN4] L. Arkeryd and A. Nouri, On the stationary Povzner equation in three space variables, J. Math. Kyoto Univ. 39 (1999), 115-153.

[AN5] L. Arkeryd and A. Nouri, On the Milne problem and the hydrodynamic limit for a steady Boltzmann equation model, J. Stat. Phys. 99 (2000), 993-1019.

[AN6] L. Arkeryd and A. Nouri, $L^{1}$ solutions to the stationary Boltzmann equation in a slab, Ann. Fac. Sci. Toulouse Math. 9 (2000), 375-413.

[AN7] L. Arkeryd and A. Nouri, The stationary Boltzmann equation in $\mathbb{R}^{n}$ with given indata, Ann. Scuola Norm. Sup. Pisa 31 (2002), 1-28.

[AN8] L. Arkeryd and A. Nouri, The stationary nonlinear Boltzmann equation in a Couette setting; multiple, isolated $L^{q}$-solutions and ydrodynamic limits, submitted to J. Stat. Phys. 2004.

[AN9] L. Arkeryd and A. Nouri, A large data existenced result for the stationary Boltzmann equation in a cylindrical geometry, to appear in Ark. Mat., 2005.

[AN10] L. Arkeryd and A. Nouri, On a Taylor Couette type bifurcation from a kinetic point of view, in preparation.

[BCN1] C. Bardos, R. E. Caflish and B. Nicolaenko, Thermal layer solutions of the Boltzmann equation, Progr. Phys. 10, Birkhäuser, Boston, 1985, 235-251.

[BCN2] C. Bardos, R. E. Caflish and B. Nicolaenko, The Milne and Kramers problems for the Boltzmann equation of a hard spere gas, Comm. Pure Appl. Math. 39 (1986), 323-352.

[BGL] C. Bardos, F. Golse and C. D. Levermore, Fluid dynamic limits of kinetic equations I, J. Stat. Phys. 63 (1991), 323-344.

[BU] C. Bardos and S. Ukai, The classical incompressible Navier-Stokes limit of the Boltzmann equation, Math. Mod. Meth. Appl. Sci. 1 (1991), 235-257.

[CCW] E. Carlen, M. Carvalho and B. Wennberg, Entropic convergence for solutions of the Boltzmann equation with general physical initial data, Transport Th. Stat. Phys. 26 (1997), 373-378.

[CGT] E. Carlen, E. Gabetta and G. Toscani, Propagation of smoothness and the rate of exponential convergence to equilibrium for a spatially homogeneous Maxwellian gas, Comm. Math. Phys. 199 (1999), 521-546. 
[C1] C. Cercignani, Half-space problems in the kinetic theory of gases, in: Trends in Applications of Pure Mathematics to Mechanics, Springer, Berlin, 1986, 35-50.

[C2] C. Cercignani, The Boltzmann Equation and its Applications, Appl. Math. Sci. 67, Springer, 1988.

[CIP] C. Cercignani, R. Illner and M. Pulvirenti, The Mathematical Theory of Dilute Gases, Springer, Berlin, 1994.

[DEL] A. De Masi, R. Esposito and J. L. Lebowitz, Incompressible Navier-Stokes and Euler limits of the Boltzmann equation, Comm. Pure Appl. Math. 42 (1989), 1189-1214.

[D] L. Desvillettes, Sur quelques hypothèses nécessaires à l'obtention du développement de Chapman-Enskog, preprint 1994.

[DPL] R. J. DiPerna and P. L. Lions, On the Cauchy problem for Boltzmann equations: Global existence and weak stability, Ann. Math. 130 (1989), 321-366.

[ELM1] R. Esposito, J. L. Lebowitz and R. Marra, Hydrodynamic limit of the stationary Boltzmann equation in a slab, Comm. Math. Phys. 160 (1994), 49-80.

[ELM2] R. Esposito, J. L. Lebowitz and R. Marra, The Navier-Stokes limit of stationary solutions of the nonlinear Boltzmann equation, J. Stat. Phys. 78 (1995), 389-412.

[EP] R. Ellis and M. Pinsky, The first and second fluid approximations to the linearized Boltzmann equation, J. Math. pures appl. 54 (1975), 125-156.

[GPS] F. Golse, B. Perthame and C. Sulem, On a boundary layer problem for the nonlinear Boltzmann equation, Arch. Rat. Mech. Anal., 103 (1988), 81-96.

[GP] F. Golse and F. Poupaud, Stationary solutions of the linearized Boltzmann equation in a half space, Math. Meth. Appl. Sci. 11 (1989), 483-502.

[G1] H. Grad, Asymptotic theory of the Boltzmann equation I, Phys. Fluids 6 (1963), 147181.

[G2] H. Grad, Asymptotic theory of the Boltzmann equation II, in: Rarefied Gas Dynamics, Acad. Press, NY, 1963, 26-59.

[G3] H. Grad, Asymptotic theory of the Boltzmann equation, Phys. Fluids 6 (1963), 147181.

[Gu] J. P. Guiraud, Problème aux limites intérieur pour l'équation de Boltzmann en régime stationnaire, faiblement non linéaire, J. Mécanique 11 (1972), 183-231.

[H] A. Heintz, On solvability of boundary value problems for the non linear Boltzmann equation in a bounded domain, in Molecular Gas Dynamics.

[K] M. N. Kogan, Rarefied Gas Dynamics, Plenum, NY, 1969.

[L] P. L. Lions, Compactness in Boltzmann's equation via Fourier integral operators and applications, J. Math. Kyoto Univ. 34 (1994), 391-427.

[M] N. Maslova, Non-Linear Evolution Equations, Kinetic Approach, Series on Advances in Mathematics for Applied Sciences 10, World Scientific, 1993.

[Mi] S. Mischler, On weak-weak convergence and applications to the initial boundary value problem for kinetic equations, preprint, Versailles, 1999.

[N] A. Nouri, On a Cauchy inequality for the Boltzmann equation, preprint, 1999.

[P] Y. P. Pao, Boundary value problems for the linearized and weakly nonlinear Boltzmann equation, J. Math. Phys. 8 (1967), 1893-1898.

[Pe] R. Pettersson, On convergence to equilibrium for the linear Boltzmann equation without detailed balance assumptions, in: Rarefied Gas Dynamics 19, Oxford UP, 1995, 107-113.

[S] Y. Sone, Kinetic Theory and Fluid Dynamics, Birkhäuser, Boston, 2002. 
[SD1] Y. Sone and T. Doi, Bifurcation of a flow of a gas between coaxial circular cylinders with evaporation and condensation II, in: Rarefied Gas Dynamics, AIAA, New York, 2002, 642-653.

[SD2] Y. Sone and T. Doi, Bifurcation of and ghost effect on the temperature field in the Bénard problem of a gas in the continuum limit, Phys. Fluids 15 (2003), 1405-1423.

[T] L. Triolo, A formal generalization of the H-theorem in kinetic theory, Report, Roma Tor Vergata, 1993.

[UA] S. Ukai and K. Asano, Steady solutions of the Boltzmann equation for a gas flow past an obstacle; I existence, Arch. Rat. Mech. Anal. 84 (1983), 249-291.

[V] C. Villani, A review of mathematical topics in collisional kinetic theory, in: Handbook of Mathematical Fluid Dynamics, Vol. I, North-Holland, Amsterdam, 2002, 71-305.

[W] B. Wennberg, B., On an entropy dissipation inequality for the Boltzmann equation, C. R. Acad. Sci. Paris 315 (1992), 1441-1446. 